\title{
A SMALL EXAMPLE OF NON-LOCAL OPERATORS HAVING NO TRANSMISSION PROPERTY
}

\author{
By
}

\author{
Yasushi IsHIKAWA
}

\begin{abstract}
We give an example of non-local pseudo-differential operators which do not have the transmission property. The symbol of this operator is given by the composition of a complete Bernstein function by a quadratic form. The study is motivated by the boundary problem of non-local operators.
\end{abstract}

\section{Introduction}

In connection with the theory of classical Markov processes and potential theory, the subordination operation of processes appears naturally in the theory of convolution semigroups (cf. e.g. [1], [20]). For example, the semigroup subordinated to a 1-dimensional Brownian semigroup $\mu_{t}$ by means of a one-sided stable semigroup $v_{t}^{\alpha}$ of index $\alpha \in(0,1)$ is a symmetric stable semigroup of index $\left.2 \alpha: \sigma_{t}=\int_{0}^{\infty} \mu_{s} d v_{t}^{\alpha}(s)([1],(9.23)-5)\right)$. In terms of "symbols" of generators for the semigroups, the above subordination operation corresponds to the composition $\sigma=f \circ \psi_{0}$ of the Bernstein function $f(\xi)=\xi^{\alpha}$ with the continuous negative definite function $\psi_{0}(\gamma)=c \gamma^{2}$. (That is, $\left(\hat{\sigma_{t}} \hat{u}\right)=e^{-t \sigma} \hat{u}$, where ${ }^{\wedge}$ denotes the Fourier transformation.) Here $\sigma$ is again a continuous negative definite function (cf. [1] (9.20)).

On the other hand, the transmission property of a linear oprator $P$ on $\boldsymbol{R}^{d}$ with respect to the boundary $\boldsymbol{R}^{d-1}$ means that $P$ is continuous $C_{0}^{\infty}\left(\overline{\boldsymbol{R}_{+}^{d}}\right) \rightarrow C^{\infty}\left(\overline{\boldsymbol{R}_{+}^{d}}\right)$, $\left.u \mapsto(P \bar{u})\right|_{\boldsymbol{R}_{+}^{d}}$, where $\bar{u}$ is the extension of $u$ to $\boldsymbol{R}^{d}$ by 0 outside $\boldsymbol{R}_{+}^{d}$. Here and in the sequel $C^{\infty}\left(\overline{\boldsymbol{R}_{+}^{d}}\right)$ denotes the space of functions in $C^{\infty}\left(\boldsymbol{R}_{+}^{d}\right)$ having continuous extensions (including derivatives of all orders) to the closure $\overline{\boldsymbol{R}_{+}^{d}}$ of $\boldsymbol{R}_{+}^{d}$, and $C_{0}^{\infty}\left(\overline{\boldsymbol{R}_{+}^{d}}\right)$

2000 Mathematics Subject Classification. Primary 47D07; Secondary 60J35.

Key words: transmission property, convolution semigroup, Bernstein function

Received June 15, 2000.

Revised October 30, 2000. 
denotes the space of functions in $C^{\infty}\left(\overline{\boldsymbol{R}_{+}^{d}}\right)$ having compact supports in the relative topology.

The property of transmission appears naturally in various boundary value problems, such as the existence of the Feller semigroup in a bounded domain with boundary conditions. For example, the existence of the "sectional trace" $\left.P u\right|_{\partial D}$ of $P u$ (where $P$ denotes the infinitesimal generator of the semigroup, which appears to be an integro-differential operator of second order) on the boundary $\partial D$ plays an important role in constructing the Feller semigroup in $D \cong \boldsymbol{R}_{+}^{d}$ (cf. [6] Theorem (4.6) and Introduction p. 548, [13] Section 1, [21] p. 22, see also pp. 5-10). Here the transmission property of $P$ with respect to the boundary (from inside) is naturally assumed.

However, it is known that the pseudo-differential operator $\sigma(D)$ induced by the above negative definite symbol $\sigma$ (i.e.,

$$
\sigma(D) u(x)=\int_{\boldsymbol{R}^{d}} e^{i x \cdot \gamma} \sigma(\gamma) \hat{u}(\gamma) \bar{d} \gamma, \quad \sigma=f \circ \psi_{0}
$$

$\left.\bar{d} \gamma=(1 / 2 \pi)^{d} d \gamma\right)$ does not satisfy this property in general (see [12] for the examples). This suggests in particular that we should restrict the types of symbols of infinitesimal generators so that the boundary condition is well defined in constructing the Feller semigroup. A more precise study is done in [19] on the relation between the regularity of the function related with the infinitesimal generator at $x_{0} \in D$ near the boundary $\partial D$, and the number of such jumps of underlying process that have the directions of exiting $D$ from $x_{0}$.

The aim of this note is to give an (counter-) example of a certain type of general negative definite symbols for which the corresponding pseudo-differential operator, although it has a proper probabilistic meaning, does not satisfy the transmission property. Namely, we will show that the variable-coefficient pseudodifferential operator $\sigma(x, D)$ having the symbol $\sigma(x, \gamma)$, which is the composition of a general continuous negative definite function $\psi(x, \gamma)$ of quadratic type with a complete Bernstein function $f(\xi)$, does not have the transmission property (Theorems 2.3 and 3.3). Although it is suspected that most non-local operators may not have the transmission property in general, this result may provide a small but concrete example of such operators.

The motivation for our study is the close relation between the transmission property and the operators induced by general negative definite functions which generate Feller semigroups under boundary conditions (cf. [3], [14]; see also [2]Introduction for a probabilistic interpretation). That is, the transmission property of the operator $\sigma(x, D)$ to some direction is related to the (micro-) analyticity of 
the symbol $\gamma \mapsto \sigma(x, \gamma)$ to the corresponding direction. This relation is studied in a simple case in [12]. Our result should be interesting since (1) according to $\mathrm{Ph}$. Courrège [7], the generator of a Feller semigroup is a pseudo-differential operator $\sigma(x, D)$ for which the symbol $\sigma(x, \xi)$ is a general negative definite symbol, and since (2) as we have noticed above, the subordination operation appears quite naturally in the theory of Markov processes (cf. e.g., [15]).

\section{Acknowledgement}

The author would like to thank the anonymous referee for the careful reading of the first version of this article, and for giving useful comments which helped to improve it significantly.

\section{Preliminaries}

A function $\psi: \boldsymbol{R}^{d} \rightarrow \boldsymbol{C}$ is said to be negative definite if for all $n \in \boldsymbol{N}$ and all $\left(\gamma^{1}, \ldots, \gamma^{n}\right) \in \boldsymbol{R}^{d} \times \cdots \times \boldsymbol{R}^{d}$, matrix $\left(\psi\left(\gamma^{i}\right)+\overline{\psi\left(\gamma^{j}\right)}-\psi\left(\gamma^{i}-\gamma^{j}\right)\right)$ is positive Hermitian, where $\overline{\psi(\gamma)}$ denotes the complex conjugate of $\psi(\gamma)$. We call a function $\psi: \boldsymbol{R}^{d} \times \boldsymbol{R}^{d} \rightarrow \boldsymbol{C}$ a general negative definite function if it is continuous and for each $x$ the function $\gamma \mapsto \psi(x, \gamma)$ is negative definite. For example, the "symbol" of a differential operator of uniformly elliptic type on $\boldsymbol{R}^{d}: \sum_{i, j=1}^{d} a^{i j}(x) D_{i} D_{j}$ where $a^{i j} \in C^{\infty}\left(\boldsymbol{R}^{d}\right)$, is a general negative definite function $\psi_{0}(x, \gamma)=\sum_{i, j=1}^{d} a^{i j}(x) \gamma_{i} \gamma_{j}$. A $C^{\infty}$-function $f:(0, \infty) \rightarrow \boldsymbol{R}$ is said to be a Bernstein function if $f \geq 0$ and $(-1)^{p} D^{p} f \geq 0$ for all integers $p \geq 1$. A Bernstein function $f(\xi)$ has a (canonical) integral representation

$$
f(\xi)=a+b \xi-\int_{0}^{\infty}\left(e^{-\xi \cdot s}-1\right) d \mu(s)
$$

where $a, b \geq 0$ and $d \mu$ is a positive measure satisfying $\int_{0}^{\infty} s /(1+s) d \mu(s)<\infty$. (cf. [1] (9.8)). A Bernstein function $f$ is said to be a complete Bernstein function if the measure $\mu$ above is absolutely continuous with respect to Lebesgue measure on $(0, \infty)$. We assume further it is non-degenerate, that is, for some $n_{0} \in \boldsymbol{N}$ and $C>0$,

$$
d \mu(s) \geq C \frac{d s}{(1+s)^{n_{0}}} \quad \text { on }(0,+\infty)
$$

where $d s$ denotes the Lebesgue measure on $(0,+\infty)$.

For a given complete Bernstein function, we can make a composition of $f$ with the quadratic form $\psi_{0}$ above, which we write by $\sigma: \sigma(x, \gamma) \equiv\left(f \circ \psi_{0}\right)(x, \gamma)$. 
This again consitutes a (continous) general negative definite function ([1] (9.20)). The symbol $\sigma(x, \gamma)$ induces the pseudo-differential operator $\sigma(x, D)$ on $\boldsymbol{C}_{0}^{\infty}\left(\boldsymbol{R}^{d}\right)$ (and on $\mathscr{S}^{\prime}\left(\boldsymbol{R}^{d}\right)$ ) by

$$
\begin{aligned}
\sigma(x, D) u & =\int_{\boldsymbol{R}^{d}} e^{i x \cdot \gamma} \sigma(x, \gamma) \hat{u}(\gamma) \bar{d} \gamma \\
& =(2 \pi)^{-d / 2} \int_{\boldsymbol{R}^{d}} e^{i x \cdot \gamma} \sigma(x, \gamma) \hat{u}(\gamma) d \gamma
\end{aligned}
$$

(cf. [14] Theorem 1.2, [15] Section 4).

In effect, $\sigma(x, \gamma)=f \circ \psi_{0}(x, \gamma)$ is in the symbolclass $S_{1,0}^{2}$ of pseudo-differential operators if $f$ satisfies

$$
\left|\frac{f^{(k+1)}(s)}{f^{(k)}(s)}\right| \leq \frac{k+1}{s}, \quad s>0
$$

for $k=0,1,2, \ldots$ Here the condition (1.2) holds for all complete Bernstein functions. For the reference on these facts, see [15] Lemma 2.10.

We begin with the case $d=1$ (with the variable $x=x_{d}$ for the interpretation after). For this purpose we state below a general theory shortly, and introduce (a kind of) Hardy spaces of symbols due to [4].

Let $H$ be the vector space of all complex valued functions $f(t)$ on $\boldsymbol{R} \backslash\{0\}$ which are $C^{\infty}$ and have a regular pole at infinity. That is, the extension of $f$ (to $C)$, also denoted by $f$, satisfies that

$$
(z+1)^{p} f\left(\frac{1}{i} \frac{1-z}{1+z}\right)
$$

is a $C^{\infty}$ function on the unit circle $|z|=1$ for some integer $p$. (Note that $t=$ $(1-z) /(1+z)$ is a linear transformation on $C$.)

The space $H$ can be interpreted as the space of $C^{\infty}$ functions $f(t)$ with the property: there exist some $p \in Z$ and complex numbers $s_{p}, s_{p-1}, \ldots$, such that for all indices $k, l$ and $n \in N$ it holds that

$$
\partial_{t}^{l}\left[t^{k} f(t)-\sum_{p-N \leq j \leq p} s_{j} t^{j+k}\right]
$$

is $O\left(|t|^{p-N-1+k-l}\right)$ as $|t| \rightarrow+\infty$.

We put $H_{-1}=\{f \in H ;(1.3)$ holds with $p=-1\}$. Let $H_{-1}^{+}$(resp. $H_{-1}^{-}$) be the subspace of $H_{-1}$ consisting of those which can be extended analytically to the lower (upper) half plane and vanish at infinity. Then $H$ is the direct sum

$$
H=H_{-1}^{+}+H_{-1}^{-}+C[t],
$$


where $C[t]$ is the space of polynomials in $t$ (cf. [9] Proposition 2.2.2). We denote

$$
H^{+}=H_{-1}^{+}+C[t], \quad H^{-}=H_{-1}^{-}+C[t] .
$$

Then the above sum may also be written as

$$
H=H^{+}+H^{-}, \quad H^{+} \cap H^{-}=C[t] .
$$

Further we have a characterization of the space $H^{+}\left(\right.$resp. $\left.H^{-}\right)$:

Proposition 1.1 (Boutet de Monvel [4] (1.9)). $H^{+}$(resp. $H^{-}$) is the space of Fourier transforms of functions $\varphi(x)$ which vanish for $x<0$ (resp. $x>0$ ) and are in $C^{\infty}\left(\overline{\boldsymbol{R}}_{+}\right)$(resp. $C^{\infty}\left(\overline{\boldsymbol{R}}_{-}\right)$), rapidly decreasing at infinity for $x>0$ (resp. rapidly decreasing at infinity for $x<0)$. That is, every derivative tends to zero at infinity faster than any power of $x$ and has a limit when $x \rightarrow+0$ (resp. $x \rightarrow-0)$. Here we put $\boldsymbol{R}_{+}=(0, \infty)$ and $\boldsymbol{R}_{-}=(-\infty, 0)$.

We remark here that the space $\boldsymbol{C}[t]$ corresponds, through the inverse Fourier transform, to "polynomials" $\sum c_{k} \delta^{(k)}$ where $\delta^{(k)}=D_{x}^{k} \delta$. That is, a (sub-) space of distributions supported on $\{0\}$.

For 1-dimensional operator $\sigma(x, D)$ associated to the symbol $\sigma(x, \gamma) \in S_{1,0}^{m}$, we always have the decomposition

$$
\sigma(x, D)=\sigma_{0}(x, D)+\left(\sigma-\sigma_{0}\right)(x, D)
$$

where $\sigma$ is properly supported and $\sigma-\sigma_{0}$ has a $C^{\infty}$ distribution kernel [17] Chapter 3. (1.4)). Hence we have only to treat those symbols which correspond to properly supported operators modulo $C^{\infty}$-functions.

Let $\sigma(x, \gamma)$ be the symbol of a pseudo-differential operator $\sigma(x, D)$ which is properly supported. The support of $\gamma \mapsto \sigma(x, \gamma)$ can be decomposed into a locally finite sum: $\operatorname{supp} \sigma=\bigcup_{\lambda \in \Lambda} E_{\lambda}$, each $E_{\lambda}$ is compact in $\boldsymbol{R}$. Regarding $\sigma$ as a hyperfunction, we can decompose $\sigma$ as $\sigma=\sum_{\lambda \in \Lambda} \sigma_{\lambda}$ where supp $\sigma_{\lambda} \subset E_{\lambda}$ (cf. [16] Lemma 1.4.4). To verify the transmission property of $\sigma(x, D)$, we can assume that each symbol $\sigma(x, \gamma)$ has a compact support with respect to $\gamma$ (and hence it vanishes at infinity). This is because the operator $\sigma=\sum_{\lambda} \sigma_{\lambda}$ has the transmission property if and only if all $\sigma_{\lambda}$ 's have the transmission property (cf. [12]). This means, in view of $(1.5)^{\prime}$ of [4], we may assume the symbol $\gamma \mapsto \sigma(x, \gamma) \in H$ can be decomposed as $\sigma(x, \gamma)=h^{+} \sigma(x, \gamma)+h^{-} \sigma(x, \gamma)$, where $h^{+} \sigma(x, \gamma)=\lim _{\varepsilon \rightarrow 0+}(-1 / 2 \pi i) \int_{-\infty}^{+\infty}\left(\sigma\left(x, \gamma^{\prime}\right) /\left(\gamma^{\prime}-\gamma+i \varepsilon\right)\right) d \gamma^{\prime}$ and $h^{-} \sigma(x, \gamma)=$ $\lim _{\varepsilon \rightarrow 0+}(1 / 2 \pi i) \int_{-\infty}^{+\infty}\left(\sigma\left(x, \gamma^{\prime}\right) /\left(\gamma^{\prime}-\gamma-i \varepsilon\right)\right) d \gamma^{\prime}$, with $h^{+} \sigma \in H^{+}, h^{-} \sigma \in H^{-}$for each $x$. We have the following 
LEMMA 1.2. Let $x \in \boldsymbol{R}$. If $\gamma \mapsto \tilde{\sigma}(x, \gamma)$ is in $H^{+}$(resp. $\left.H^{-}\right)$then the distribution kernel $\tilde{g}(x, y)$ of $\tilde{\sigma}(x, D)$ vanishes for $y<x$ (resp. $y>x)$ and is in $C^{\infty}\left(\overline{\boldsymbol{R}}_{+}\right)$ (resp. in $C^{\infty}\left(\overline{\boldsymbol{R}}_{-}\right)$) rapidly decreasing at infinity for $y>x$ (resp. $y<x$ ); and the converse holds.

Proof. This lemma follows from Proposition 1.1 in view of

$$
\tilde{g}(x, y)=\int e^{i y \cdot \gamma} \tilde{\sigma}(x, \gamma) \bar{d} \gamma
$$

and the shift $y \rightarrow y-x$. That is, we have

$$
\begin{aligned}
\tilde{\sigma}(x, D) f(x) & =\iint e^{i\left(x-y^{\prime}\right) \cdot \gamma} \tilde{\sigma}(x, \gamma) f\left(y^{\prime}\right) d y^{\prime} \bar{d} \gamma \\
& =\iint e^{i\left(x-y^{\prime}\right) \cdot \gamma} \tilde{\sigma}(x, \gamma) \bar{d} \gamma f\left(y^{\prime}\right) d y^{\prime} .
\end{aligned}
$$

We write above as

$$
\tilde{\sigma}(x, D) f(x)=\int g\left(x, x-y^{\prime}\right) f\left(y^{\prime}\right) d y^{\prime} .
$$

We put $z=-y^{\prime}$. Then by Proposition 1.1 $z \mapsto g(0, z)$ vanishes for $z<0$, and is $C^{\infty}\left(\overline{\boldsymbol{R}}_{+}\right)$rapidly decreasing at infinity for $z>0$. The shift in $y^{\prime}$ by $x\left(y^{\prime} \rightarrow y^{\prime}-x\right)$ corresponds to $-y^{\prime} \rightarrow x-y^{\prime}=x+z$. Hence $x-y^{\prime}=y \mapsto \tilde{g}(x, y)=g\left(x, x-y^{\prime}\right)$ vanishes for $y<x$ and is $C^{\infty}(\{y \geq x\})$ rapidly decreasing at infinity for $y>x$ and has a limit as $y \rightarrow x+0$.

q.e.d.

\section{Case $d=1$}

In what follows in this section we shall confine ourselves to those symbols $\sigma(x, \gamma)$ which can be written as $\sigma(x, \gamma)=\left(f \circ \psi_{0}\right)(x, \gamma)$, where $\psi_{0}(x, \gamma)=c(x) \gamma^{2}$, $c(x) \in C^{\infty}(\boldsymbol{R}), c(x)>0, c(x)$ is bounded including derivatives of all orders, and $f$ is a complete Bernstein function on $(0,+\infty)$. That is, $\sigma$ has an expression

$$
\sigma(x, \gamma)=a+b c(x) \gamma^{2}-\int_{0}^{\infty}\left(e^{-c(x) \gamma^{2} \cdot s}-1\right) d \mu(s),
$$

with $a, b, d \mu(s)$ satisfying conditions in Section 1. It follows by an elementary calculation that for each $x \gamma \mapsto \sigma(x, \gamma) \in S_{1,0}^{2}$.

Let $Y=\boldsymbol{R}_{+}$or $Y=\boldsymbol{R}_{-}$. For a real valed function $u \in C^{\infty}(\bar{Y})$ we put $\bar{u}$ as $\bar{u}=u$ in $Y$ and $\bar{u}=0$ outside of $Y$. We remark that we write the extension for real $u$ as above as long as there is no fear of confusion with its complex con- 
jugate. On the other hand, we denote by the same $f$ the complex extension of $f$ when $f$ represents a Bernstein function.

We define the operator $P_{Y}$ on $C_{0}^{\infty}(\bar{Y})$ by $P_{Y} u=\left.P \bar{u}\right|_{Y}$.

DEFINITION 2.1. We say that the transmission property to the left (to the right) with respect to the origin holds for $\boldsymbol{P}$ if $\boldsymbol{P}_{Y}$ is continuous $C_{0}^{\infty}\left(\overline{\boldsymbol{R}}_{+}\right) \rightarrow C^{\infty}\left(\overline{\boldsymbol{R}}_{+}\right)$(resp. $\left.C_{0}^{\infty}\left(\overline{\boldsymbol{R}}_{-}\right) \rightarrow C^{\infty}\left(\overline{\boldsymbol{R}}_{-}\right)\right)$.

REMARK. Our definition transmission property to the left (to the right) may not coincides with the widely used ones such as transmission property with respect to the boundary or two-sided transmission property (cf. [4]). However, in connection with the underlying jump process related to the symbol $f$, our terminology may well illustrates the direction of jumps at the boundary. Our definition coincides with that in the former [12].

In view of the expressions (cf. (1.6), (1.7)

$$
\sigma(x, D) u(x)=\int u\left(y^{\prime}\right) g\left(x, x-y^{\prime}\right) d y^{\prime}
$$

we have from Lemma 1.2 and (1.4) the following

Proposition 2.2. Let $\sigma(x, D)$ be the pseudo-differencial operator with the symbol $\sigma(x, \gamma)$ given by (2.1). Then for all $i \in N \sigma_{(i)}(x, \gamma) \in H^{+}\left(\right.$resp. $\left.H^{-}\right)$in a right neighborhood of $x=0$ in $\overline{\boldsymbol{R}}_{-}$(resp. in a left neighborhood of $x=0$ in $\overline{\boldsymbol{R}}_{+}$) if and only if $\sigma(x, D)$ satisfies the transmission property to the left (to the right) with respect to the origin. Here $\sigma_{(i)}(x, \gamma)=D_{x}^{i} \sigma(x, \gamma)$.

Proof. By Green's formula (cf. [9] (2.2.38), (2.2.39))

$$
D \bar{u}=\overline{(D u)}-i u(0) \delta
$$

hence

$$
\begin{aligned}
D \sigma_{Y} u & =\left.[D(\sigma \bar{u})]\right|_{Y}=\left.\left[\sigma^{\prime} \bar{u}+\sigma D \bar{u}\right]\right|_{Y} \\
& =\left.\left[\sigma^{\prime} \bar{u}\right]\right|_{Y}+\left.[\sigma(\overline{(D u})]\right|_{Y}-i\left(\left.(\sigma \delta)\right|_{Y}\right) \cdot u(0) \\
& =\sigma_{Y}^{\prime} u+\sigma_{Y}(D u)-\left.i(g(\cdot, \cdot))\right|_{Y} \cdot u(0)
\end{aligned}
$$

where $\sigma^{\prime}=D_{x} \sigma$. Last equality follows from $\sigma\left(x^{\prime}, D\right) \delta(x)=\int \delta\left(y^{\prime}\right) g\left(x^{\prime}, x-y^{\prime}\right) d y^{\prime}=$ $g\left(x^{\prime}, x\right)$ in the sense of distributions. 
For the heiger order derivatives we have by the successive use of (2.3),

$$
D^{k} \bar{u}=\overline{\left(D^{k} u\right)}-i \sum_{0 \leq l \leq k-1}\left(D^{k-l-1} u\right)(0) D^{l} \delta
$$

Hence we can calculate

$$
\begin{aligned}
D^{k}\left(\sigma_{Y} u\right)= & \left.\left(\left(D^{k} \sigma\right) \bar{u}\right)\right|_{Y}=\left.\sum_{i=0}^{k}\left(\begin{array}{c}
k \\
i
\end{array}\right)\left(D_{x}^{i} \sigma\right)\left(D_{x}^{k-i} \bar{u}\right)\right|_{Y} \\
= & \sum_{i=0}^{k}\left\{\left(\begin{array}{c}
k \\
i
\end{array}\right)\left(D_{x}^{i} \sigma\right) \overline{\left(D_{x}^{k-i} u\right)}-i \sum_{0 \leq l \leq k-i-1}\left(\begin{array}{c}
k \\
i
\end{array}\right)\right. \\
& \left.\times\left(D_{x}^{i} \sigma\right)\left(D_{x}^{k-i-l-1} u\right)(0) D_{x}^{l} \delta\right\}\left.\right|_{Y} \\
= & \sum_{i=0}^{k}\left(\begin{array}{c}
k \\
i
\end{array}\right)\left(D_{x}^{i} \sigma\right)_{Y}\left(D^{k-i} u\right)-i \sum_{i=0}^{k} \sum_{0 \leq l \leq k-i-1}\left(\begin{array}{c}
k \\
i
\end{array}\right) \\
& \times\left.\left(D_{x}^{i} \sigma\right)\left\{\left(D^{k-i-l-1} u\right)(0) D_{x}^{l} \delta\right\}\right|_{Y} .
\end{aligned}
$$

Here

$$
\begin{aligned}
\left(D_{x}^{i} \sigma\right)\left(D_{x}^{l} \delta\right)(x) & =\int D^{l} \delta\left(y^{\prime}\right) g^{(i)}\left(x, x-y^{\prime}\right) d y^{\prime} \\
& =\int \delta\left(y^{\prime}\right) D_{y}^{l} g^{(i)}\left(x, x-y^{\prime}\right) d y^{\prime}=D_{y}^{l} g^{(i)}(x, x)
\end{aligned}
$$

where we put $g^{(i)}(x, \cdot)=\mathscr{F}^{-1}\left[\left(D_{x}^{i} \sigma\right)(x, \gamma)\right](x, \cdot)$ and $D_{y} g$ means the derivative of $g(\cdot, \cdot-y)$ with respect to $y$. Hence R.H.S. of $(2.5)=$

$$
\sum_{i=0}^{k}\left(\begin{array}{c}
k \\
i
\end{array}\right)\left(D_{x}^{i} \sigma\right)_{Y}\left(D^{k-i} u\right)-i \sum_{i=0}^{k} \sum_{0 \leq l \leq k-i-1}\left(\begin{array}{c}
k \\
i
\end{array}\right) D_{y}^{l} g^{(i)}(\cdot, \cdot) \cdot\left(D^{k-i-l-1} u\right)(0)
$$

Further we have in the first term above

$$
\left(D_{x}^{i} \sigma\right)_{Y}\left(D^{k-i} u\right)(x)=\left.\int \overline{\left(D^{k-i} u\right)}\left(y^{\prime}\right) g^{(i)}\left(x, x-y^{\prime}\right) d y^{\prime}\right|_{Y}
$$

We choose $Y=\boldsymbol{R}_{-}$below for simplicity. If for all $i \in \boldsymbol{N} \gamma \mapsto \sigma_{(i)}(x, \gamma) \in H^{+}$, then by Lemma 1.2 and $(2.6)$ we have $\left(\left(\sigma_{(i)}\right)_{\boldsymbol{R}_{-}}\right) u \in C^{\infty}\left(\overline{\boldsymbol{R}}_{-}\right)$for all $u \in C_{0}^{\infty}\left(\overline{\boldsymbol{R}}_{-}\right)$. 
Since each $g^{(i)}(x, y)$ is $C^{\infty}$ outside of the diagonal set $\{x=y\}$ (pseudo-local property), this implies that $\left(\sigma_{\boldsymbol{R}_{-}}\right) u$ is in $C^{\infty}\left(\overline{\boldsymbol{R}}_{-}\right)$for all $u \in C_{0}^{\infty}\left(\overline{\boldsymbol{R}}_{-}\right)$if $\left.D_{y}^{l} g^{(i)}(\cdot, \cdot)\right|_{\boldsymbol{R}_{-}}$ is in $C^{\infty}\left(\overline{\boldsymbol{R}}_{-}\right)$for all $l, i=0, \ldots, k ; k=1,2, \ldots$ By Lemma 1.2 this holds if $\gamma \mapsto$ $\sigma_{(i)}(x, \gamma) \in H^{+}$for all $i=0,1,2, \ldots$

On the other hand, if for some $i \in N \gamma \mapsto \sigma_{(i)}(x, \gamma) \notin H^{+}$, then by Lemma 1.2 the corresponding $g^{(i)}(x, x-\cdot)$ does not satisfy the property to be in $C^{\infty}\left(\overline{\boldsymbol{R}}_{-}\right)$. Then, in (2.6), we can find $\tilde{u} \in C^{\infty}\left(\boldsymbol{R}_{-}\right)$which is $m$-flat for $m<k-i$ but is not $(k-i)$-flat in a (relatively closed) left neighborhood of $y^{\prime}=0$. [Here we say $v$ is $m$-flat at $y^{\prime}$ if $D^{j} v\left(y^{\prime}\right)=0$ for all $j$ with $j \leq m$.] By Lemma 1.5.2 of [18] we can approximate $\tilde{u}$ by $u \in C^{\infty}\left(\overline{\boldsymbol{R}}_{-}\right)$up to the $(k-i-1)$-norm in a (relatively closed) left neighborhood of $y^{\prime}=0$. Since $k \in N$ is arbitrary, for some $u \in$ $C_{0}^{\infty}\left(\overline{\boldsymbol{R}}_{-}\right),\left(\sigma_{\boldsymbol{R}_{-}}\right) u$ is not in $C^{\infty}\left(\overline{\boldsymbol{R}}_{-}\right)$by the above decomposition. This concludes the assertion.

q.e.d.

Now we state our main result.

THEOREM 2.3. The pseudo-differential operator $\sigma(x, D)$ having the symbol $\sigma(x, \gamma) \equiv\left(f \circ \psi_{0}\right)(x, \gamma)$ given by (2.1), such that $d \mu \neq \equiv$ and $f$ satisfy (1.1), does not have the transmission property to either side with respect to the origin.

Proof. Recall the canonical representation of $f$ :

$$
f(\xi)=a+b \xi-\int_{0}^{\infty}\left(e^{-\xi \cdot \zeta}-1\right) d \mu(\zeta) .
$$

We assume $d \mu \not \equiv 0$ and

$$
d \mu(s) \geq C \frac{d s}{(1+s)^{n_{0}}}
$$

on $(0,+\infty)$.

It is well known (cf. [1] (9.12)) that the Bernstein function $f(\xi)$ has the canonical holomorphic extension to the open half plane $\operatorname{Re} \xi>0$, which we denote by the same $f$, such that

$$
f(\xi+i \eta) \in \mathcal{O}\{\xi>0\}
$$

(Further $f(\xi+i \eta) \in C\{\xi \geq 0\}$ if and only if $\lim _{x \rightarrow 0} f(x)<\infty$.) However we have by (2.7) that for all $\varepsilon>0$

$$
f(\xi+i \eta) \notin \mathcal{O}\{\xi>-\varepsilon\} .
$$


Writing $\sigma(x, \gamma)=a+b c(x) \gamma^{2}-\int_{0}^{\infty}\left(e^{-c(x) \gamma^{2} \cdot s}-1\right) d \mu(s) \quad((2.1))$ and putting $\gamma=$ $\gamma_{1}+i \gamma_{2}$, we have

$$
\sigma\left(x, \gamma_{1}+i \gamma_{2}\right)=a+b(x)\left\{\left(\gamma_{1}^{2}-\gamma_{2}^{2}\right)+2 i \gamma_{1} \gamma_{2}\right\}-\int_{0}^{\infty}\left(e^{-c(x)\left\{\left(\gamma_{1}^{2}-\gamma_{2}^{2}\right)+i 2 \gamma_{1} \gamma_{2}\right\} \cdot s}-1\right) d \mu(s),
$$

and we find by (2.8), (2.7) that $\gamma \mapsto \sigma(x, \gamma)$ has a holomorphic extension to $\left\{\gamma_{1}+i \gamma_{2} ; \gamma_{1}^{2}>\gamma_{2}^{2}\right\}$, but not to $\left\{\gamma_{1}+i \gamma_{2} ;(1+\varepsilon)^{2} \gamma_{1}^{2} \geq \gamma_{2}^{2}\right\}$ for any $\varepsilon>0$.

Hence $\sigma(x, \gamma)$ belongs neither to $H^{+}$nor to $H^{-}$properly in a left or right neighborhood of $x=0$ in $\bar{Y}$ respectively. This means $\gamma \mapsto \sigma(x, \gamma)$ contains both components of $H_{-1}^{+}$and $H_{-1}^{-}$in a left or right neighborhood of $x=0$ in $\bar{Y}$. By Proposition 2.2, $\sigma(x, D)$ does not have the transmission property to either side.

q.e.d.

\section{General Case}

Let $X=\boldsymbol{R}^{d}, Y=\boldsymbol{R}_{+}^{d}=\left\{x \in \boldsymbol{R}^{d} ; x_{d}>0\right\}$ (resp. $Y=\boldsymbol{R}_{-}^{d}=\left\{x \in \boldsymbol{R}^{d} ; x_{d}<0\right\}$ ). For $u \in C^{\infty}(\bar{Y})$, we put $\bar{u}$ similarly as in Section $2: \bar{u}=u$ in $Y$ and $\bar{u}=0$ outside of $Y$. Let $P$ be a pseudo-differential operator on $Y$. We put $P_{Y}$ by $P_{Y} u=\left.P \bar{u}\right|_{Y}$. We denote the space $\boldsymbol{R}^{d}=\boldsymbol{R}^{d-1} \times \boldsymbol{R}$, and accordingly we write $x=\left(x^{\prime}, x_{d}\right)$ and $\gamma=\left(\gamma^{\prime}, \gamma^{d}\right)$.

DEFINITION 3.1. We say that the transmission property with respect to $Y$ for $P$ holds for $Y$ if $P_{Y}$ is continuous $C_{0}^{\infty}(\bar{Y}) \rightarrow C^{\infty}(\bar{Y})$

Let $\sigma(x, D)$ be the pseudo-differential operator on $X$ with the symbol $\sigma(x, \gamma) \in$ $S_{1,0}^{2}$ given by the formula above (1.2). We consider the partial distribution kernel (partial Fourier inversion) $\mathscr{F}_{\gamma_{d} \rightarrow y_{d}}^{-1} \sigma(x, \gamma)=(1 / 2 \pi) \int e^{i y_{d} \gamma_{d}} \sigma(x, \gamma) d \gamma_{d}$. That is, we decompose $\sigma(x, D) u(x)$ as follows:

$$
\begin{aligned}
& \sigma(x, D) u(x)=\iint e^{i\left(x-y^{\prime}\right) \cdot \gamma} \sigma(x, \gamma) u\left(y^{\prime}\right) d y^{\prime}(1 / 2 \pi)^{d} d \gamma \\
& =\int_{\boldsymbol{R}^{d-1}} \int_{\boldsymbol{R}^{d-1}} e^{i\left(x_{1}-y_{1}^{\prime}, \ldots, x_{d-1}-y_{d-1}^{\prime}\right) \cdot\left(\gamma_{1}, \ldots, \gamma_{d-1}\right)} \\
& \quad \times\left\{\iint^{i\left(x_{d}-y_{d}\right) \cdot \gamma_{d}} \sigma(x, \gamma) d y_{d}^{\prime} \bar{d} \gamma_{d} u\left(y_{1}, \ldots, y_{d-1}, y_{d}\right)\right\} d y_{1}^{\prime} \cdots d y_{d-1}^{\prime} \bar{d} \gamma_{1} \cdots \bar{d} \gamma_{d-1} \\
& =\int_{\boldsymbol{R}^{d}} \int_{\boldsymbol{R}^{d-1}} e^{i\left(x_{1}-y_{1}^{\prime}, \ldots, x_{d-1}-y_{d-1}^{\prime}\right) \cdot\left(\gamma_{1}, \ldots, \gamma_{d-1}\right)} \\
& \quad \times\left\{\int e^{i\left(x_{d}-y_{d}^{\prime}\right) \cdot \gamma_{d}} \sigma(x, \gamma) \bar{d} \gamma_{d}\right\} u\left(y_{1}, \ldots, y_{d}\right) d y_{1}^{\prime} \cdots d y_{d}^{\prime} \bar{d} \gamma_{1} \cdots \bar{d} \gamma_{d-1} .
\end{aligned}
$$


We may write $\{\cdots\}$ as a kernel $g\left(x, x_{d}-y_{d}^{\prime} ; \gamma_{1}, \ldots, \gamma_{d-1}\right)$. This gives the partial distribution kernel

$$
\mathscr{F}_{\gamma_{d} \rightarrow y_{d}}^{-1} \sigma(x, \gamma)=\int e^{i y_{d} \gamma_{d}} \sigma(x, \gamma) \bar{d} \gamma_{d}
$$

as a function of variables $x_{1}, \ldots, x_{d}, \gamma_{1}, \ldots, \gamma_{d-1}$ and $y_{d}$.

In effect, we can reduce the case with the help of following general result to the situation in one-variable $\gamma_{d}$ with constant coefficient with respect to $x_{d^{-}}$ direction.

Proposition 3.2 (Grubb and Hörmander [8], Theorem 1.6 (i), (v)). Let $\sigma(x, \gamma) \in S_{1,0}^{m}, m \in \boldsymbol{R}$. The following two conditions are equivalent:

(a) $\sigma(x, D)$ has the transmission property with respect to $Y$

(b) For all $\alpha^{\prime} \in N^{d-1}$ and $\beta_{d} \in N$

$$
\left.\mathscr{F}_{\gamma_{d} \rightarrow y_{d}}^{-1} \sigma_{(\tilde{\beta})}^{\left(\tilde{\alpha}^{\prime}\right)}\left(x^{\prime}, 0,0, \gamma_{d}\right)\right|_{Y} \in C^{\infty}(\bar{Y}) \text {. }
$$

Here $\tilde{\alpha}^{\prime}=\left(\alpha_{1}, \ldots, \alpha_{d-1}, 0\right) \in \boldsymbol{N}^{d}, \tilde{\beta}=\left(0, \ldots, 0, \beta_{d}\right) \in \boldsymbol{N}^{d}$.

Hence by Lemma 1.2, we have only to see if, for each $x^{\prime} \in \boldsymbol{R}^{d-1}$, the function $\gamma_{d} \mapsto \sigma\left(x^{\prime}, 0,0, \gamma_{d}\right)$ is not in $H^{+}\left(\right.$resp. $\left.H^{-}\right)$. Here we have our main

THEOREM 3.3. Given a complete Bernstein function

$$
f(\xi)=a+b \xi-\int_{0}^{\infty}\left(e^{-\xi \cdot s}-1\right) d \mu(s),
$$

and a quadratic form $\psi_{0}(x, \gamma)=\sum_{i, j=1}^{d} a^{i j}(x) \gamma_{i} \gamma_{j}$, we assume that (1.1) holds with some $d \mu \not \equiv 0$, and that $a^{i j} \in C^{\infty}\left(\boldsymbol{R}^{d}\right), a_{i j}$ are bounded including derivatives of all orders, $a^{i j}=a^{j i}$, and $\left(a^{i j}(x)\right) \geq c I$ for some $c>0$. Then the pseudo-differential operator $\sigma(x, D)$ having the symbol $\sigma(x, \gamma) \equiv\left(f \circ \psi_{0}\right)(x, \gamma)$ has the transmission property with respect neither to $\boldsymbol{R}_{+}^{d}$ nor to $\boldsymbol{R}_{-}^{d}$.

Proof. Let $Y=\boldsymbol{R}_{+}^{d}$ or $\boldsymbol{R}_{-}^{d}$. The composition $\sigma \equiv f \circ \psi_{0}$ reads

$$
\sigma(x, \gamma)=a+b \sum_{i, j} a^{i j}(x) \gamma_{i} \gamma_{j}-\int_{0}^{\infty}\left(e^{-s \cdot \sum_{i, j} a^{i j}(x) \gamma_{i} \gamma_{j}}-1\right) d \mu(s) .
$$

Then we see $\sigma(x, \gamma) \in S_{1,0}^{2}$. Observe that, writing $\gamma_{d}=\gamma_{d}+i \delta_{d}$,

$$
\begin{aligned}
\sigma\left(x^{\prime}, 0,0, \gamma_{d}\right)= & a+b\left\{a^{d d}\left(x^{\prime}, 0\right)\left(\gamma_{d}^{2}-\delta_{d}^{2}\right)+2 i a^{d d}\left(x^{\prime}, 0\right) \gamma_{d} \delta_{d}\right\} \\
& -\int\left(\exp \left[-s \cdot\left\{a^{d d}\left(x^{\prime}, 0\right)\left(\gamma_{d}^{2}-\delta_{d}^{2}\right)+2 i a^{d d}\left(x^{\prime}, 0\right) \gamma_{d} \delta_{d}\right\}\right]-1\right) d \mu(s) .
\end{aligned}
$$


The non-degeneracy condition for the hermitian matrix $\left(a^{i j}\right)$ assures $a^{d d}>0$, hence again (as in Theorem 2.3) we observe $\sigma\left(x^{\prime}, 0,0, \gamma_{d}\right)$ has a holomorphic extension only to $\left\{\gamma_{d}+i \delta_{d} ; \gamma_{d}^{2}>\delta_{d}^{2}\right\}$ for each $x^{\prime} \in \boldsymbol{R}^{d-1}$.

For each $x^{\prime} \in \boldsymbol{R}^{d-1}, \sigma\left(x^{\prime}, 0,0, \gamma_{d}\right)$ belongs to $H$, and neither to $H^{+}$nor to $H^{-}$ properly, hence $\sigma(x, D)$ does not have the transmission property with respect to $Y$ by Propositions 2.3 and 3.2 ((b) is violated). q.e.d.

\section{References}

[1] Berg, C. and Forst, G., Potential theory on locally compact Abelian groups, Ergeb. Math. Grenzgeb. II. 87. Springer-Verlag, Berlin-Heidelberg-New York 1975.

[2] Bismut, J.-M., The calculus of boundary processes, Ann. Sci. École Norm. Sup. 17 (1984), 507-622.

[3] Bony, J.-M., Courrège, P. and Priouret, P., Semi-group de Feller sur une varaété à bord compact et problèmes aux limites intégro-différentiels du second ordre donnant lieu au principle du maximum, Ann. Inst. Fourier (Grenoble) 18 (1965), 369-521.

[4] Boutet de Monvel, L., Boundary problems for pseudo-differential operators, Acta Math. 126 (1971), 11-51.

[ 5 ] Boutet de Monvel, L., Comportement d'un opérateur pseudo différentiel sur une variété à bord, J. Anal. Math. 17 (1966), 241-304.

[6] Cattiaux, P., Stochastic calculus and degenerate boundary value problems, Ann. Inst. Fourier (Grenoble) 43 (1992), 541-624.

[7] Courrège, $\mathrm{Ph}$., Sur la forme intégro-différentielle des opératéurs de $C_{K}^{\infty}$ dans $C$ satisfaisant au principle du maximum, Sem. Theorie du Potentiel (1965/66) exposé 2, (M. Brelot, G. Choquet and J. Deny, eds.), Faculté des Sciences de Paris.

[ 8 ] Grubb, G. and Hörmander, L., The transmission property, Math. Scand. 67 (1990), 237-289.

[9] Grubb, G., Functional calculus of pseudo-differential boundary problems (second edition), Progress in Mathematics 65, Birkhauser, Boston, 1996.

[10] Hoh, W. and Jacob, N., Pseudo differential operators, Feller semigroups and the martingale problem, in Stochastic processes and optimal control (Friedrichroda, 1992), 95-103, Stochastic Monographs 7, Gordon and Breach, Montreux 1993.

[11] Hoh, W. and Jacob, N., On the Dirichlet problem for pseudo differential operators generating Feller semigroups, J. Funct. Anal. 137 (1996), 19-48.

[12] Ishikawa, Y., Remarks on transmission, antitransmission and antilocal properties of stable generators, Tsukuba J. Math. 12 (1988), 477-487.

[13] Ishikawa, Y., A remark on the existence of a diffusion process with non-local boundary conditions, J. Math. Soc. Japan 42 (1990), 171-184.

[14] Jacob, N., Feller semigroups, Dirichlet forms, and pseudo differential operators, Forum Math. 4 (1992), 433-446.

[15] Jacob, N. and Schilling, René L, Subordination in the sense of S. Bochner-an approach through pseudo-differential operators, Math. Nachr. 178 (1996), 199-231.

[16] Kaneko, A., Introduction to the theory of Hyperfunctions, Kluwer Academic Publishers, Dordrecht 1988.

[17] Kumano-go, H., Pseudo-differential operators (in Japanese), Iwanami, Tokyo 1974. English edition, MIT Press, Cambridge 1982.

[18] Narasimhan, R., Analysis on real and complex manifolds, Masson \& Cie, Paris 1973.

[19] Picard, J. and Savona, C., Smoothness of harmonic functions and time reversal of Markov processes, Stoch. Proc. Appl. 87 (2000), 69-91.

[20] Sato, K., Lévy processes and infinitely divisible distributions, Cambridge Univ. Press, 1999. 
[21] Taira, K., On the existence of Feller semigroups with boundary conditions, Memoirs A.M.S. 475, A.M.S., Providence 1992.

\author{
Yasushi IsHIKAwA \\ Department of Mathematics \\ Ehime University \\ Matsuyama 790-8577 (Japan) \\ e-mailslishi@math.sci.ehime-u.ac.jp
}


